\title{
Hábitos alimenticios y riesgo cardiovascular en el Corregimiento de La Florida, Risaralda 2018
}

\author{
Juan Fernando Agudelo Maya'; Bárbara Mora²; María Neyfeth Posada Morales ${ }^{3}$
}

RESUMEN Resumen: Risaralda se encuentra entre los departamentos con más prevalencia de enfermedades cardiovasculares. De ahí la importancia de establecer relación entre hábitos alimentarios y riesgo cardiovascular en población mayor de 15 años.

Métodos: estudio cuantitativo-descriptivo, observacional y transversal. Muestra no aleatoria, 186 participantes, a quienes se les aplicó test de Findrisc.

Resultados: la relación IMC (sobrepeso) tiene un RCV bajo (75\%) y un RCV alto $(25 \%)$ y con relación a la obesidad tiene un RCV bajo (84\%) y RCV alto (16\%), lo que implicaría un alto riesgo de diabetes y en consecuencia RCV alto en 10 años, dada la baja actividad física, tabaquismo, consumo excesivo en calorías, bajo consumo de frutas, leguminosas y vegetales, y poca ingesta de agua.

Conclusión: urge empoderar y sensibilizar las comunidades en estilos de vida saludable, empezando en el hogar y la escuela, ya que la población muestra tendencia hacia alimentos asociados a mayor RCV.

Enfermedades Cardiovasculares;

PALABRAS

Factores de Riesgo;

Factor de Riesgo Cardiovascular;

Riesgo Cardiovascular

Conducta Alimentaria.

1 Estudiantes Programa de Enfermería V semestre. Fundación Universitaria del Área Andina Seccional Pereira. / jagudelo28@estudiantes.areandina.edu.co*

2 bmora@areandina.edu.co

3 mposada@areandina.edu.co 


\section{Food habits and cardiovascular risk in the Corregimiento de La Florida, Risaralda 2018}

\begin{tabular}{l|l} 
ABSTRACT & $\begin{array}{l}\text { Abstract: Risaralda is among the capital municipalities with the highest } \\
\text { prevalence of cardiovascular diseases. Hence the importance of establishing } \\
\text { a relationship between eating habits and cardiovascular risk in people older } \\
\text { than } 15 \text { years. }\end{array}$
\end{tabular}

Methods: quantitative-descriptive, observational and cross-sectional study. Non-random sample, 186 participants, to whom the Findrisc test was applied.

Results: the BMI (overweight) ratio has a low CVR (75\%) and a high CVR $(25 \%)$ and in relation to obesity has a low CVR (84\%) and high CVR (16\%), which would imply a high risk of diabetes and consequently high CVR in 10 years, given the low physical activity, smoking, excessive calorie consumption, low consumption of fruits, legumes and vegetables, and low water intake. Conclusion: it is urgent to empower and sensitize communities in healthy lifestyles, starting at home and school, since the population shows a tendency towards food associated with a higher CVR.

Cardiovascular Diseases;

Risk Factors;

KEYWORDS Cardiovascular Risk Factor,

Cardiovascular Risk

Eating Behavior 


\section{INTRODUCCIÓN}

Enfermedades cardiovasculares son afecciones que afectan al sistema cardiovascular, incluyendo el corazón, vasos sanguíneos o pericardio. Dentro de los factores de riesgo se encuentran los no modificables (edad, sexo, genética, historia familiar) o los modificables, como el sedentarismo, falta de actividad física, hipertensión arterial y los hábitos alimenticios.

Estos últimos abarcan todas aquellas rutinas asociadas al acto de alimentarse, el cual si es inadecuado o se une, a otros factores de riesgo como la falta de actividad física, hábitos de fumar pueden conllevar a estados de hipertensión y diabetes, con la probabilidad de sufrir en un determinado periodo de tiempo, generalmente entre 5 a 10 años, un episodio cardiovascular (cardiopatía isquémica, enfermedad cerebrovascular, arteriopatía periférica, insuficiencia cardiaca y enfermedades de la aorta (1). De ahí que sea importante determinar el riesgo cardiovascular y empoderar al individuo para que adopte estilos de vida adecuados antes del evento patológico.

De acuerdo a la OMS (2), las enfermedades cardiovasculares (ECV), causan la muerte a 17,7 millones de personas en el mundo, y su riesgo obedece a factores comportamentales relacionados con la alimentación no saludable, ausencia de actividad física y consumo de tabaco principalmente. En América Latina, las ECV son responsables del $38 \%$ de muertes por enfermedad no transmisible y la OMS (3), las relaciona con ingesta excesiva de alcohol en mayores de 15 años de edad, consumo de sal (8.5gramos) en comparación de 4 gramos a nivel mundial y consumo por debajo de lo recomendado de frutas y verduras; lo que traduce en que América Latina tenga la más alta prevalencia en obesidad y sobrepeso, exponiéndose a ECV.

Según datos del ASIS (4), Colombia muestra una alta incidencia de muertes por ECV, asociadas también a tabaquismo, alcoholismo y sobrepeso, específicamente en el departamento de Risaralda que ha superado la tasa nacional $(64,59 \%)$ de muertes por estas enfermedades, razones que han obligado a fortalecer en esta zona las acciones que apuntan al cumplimiento de la Política Nacional de Seguridad Alimentaria y Nutricional (5), la medidas de control de la obesidad (6), la Política de Atención Integral en Salud (7) y las rutas integrales de atención en salud (8).

En general en Colombia, la población entre 18 y 64 años de edad tiene sobrepeso y la población entre 5 y 17 años de edad muestran cifras de obesidad, lo que marca tendencia al desarrollo de diabetes, hipertensión y ECV, asociadas al sedentarismo, mala alimentación y falta de educación nutricional sana ENSIN (9). En el año 2017, las personas hipertensas en el país residían en ciudades capitales, donde Pereira mostró una incidencia alta $(11,4 \%)$; y una prevalencia del 3.6 de personas diagnosticadas con diabetes mellitus (10).

Esta tendencia está caracterizada por los comportamientos socioculturales y gastronómicos de la Región Andina (11), que muestran cómo los platos típicos como 
la 'bandeja paisa', aportan en una sola comida nutricionalmente $198 \mathrm{Kcal}$, pocos vitaminas y minerales, y un alto contenido graso (colesterol $20 \mathrm{mg}$. saturados $1,9 \mathrm{~g}$, polinsaturados 4,9 g, entre otros). Igualmente, la lechona con $224 \mathrm{Kcal}$, colesterol $39 \mathrm{~g}$, saturados $2 \mathrm{~g}$, monoinsaturados $5,0 \mathrm{~g}$ y polinsaturados $4,9 \mathrm{~g}$, y el tamal con 108 Kcal, colesterol $26 \mathrm{mg}$, saturados $0,8 \mathrm{~g}$, monoinsaturados $1,3 \mathrm{~g}$ y polinsaturados 1,6 ; cuando los valores del AMDR muestran valores más bajos de ingesta, pero suficientes para mantener un buen balance calórico (12). Lo preocupante del escenario es que la mala alimentación aumenta el riesgo de ECV hasta 42 veces, ya que los altos niveles de lípidos son responsables en un 50\% de los infartos agudos del miocardio (IAM).

\section{METOLOGIA}

Tipo de estudio: descriptivo, observacional y transversal, cuyas variables buscan explorar aspectos, identificar la relación entre hábitos alimentarios y riesgo cardiovascular. El Estudio es considerado transversal, pues se mide en un tiempo determinado el fenómeno objeto de estudio.

Población y muestra: la población fue de tipo no aleatorio o por conveniencia, 186 personas que cumplieron criterios de inclusión como: ser mayor de 15 años, con antecedentes familiares de hipertensión y diabetes; entre sus antecedentes personales está el sobrepeso, obesidad, inactividad física, tabaquismo entre otros, quienes previo consentimiento o asentimiento firmado contestaron la encuesta, y se midió riesgo de sobrepeso y obesidad.

Instrumento: encuesta validada por el Instituto Departamental de Salud de Risaralda sobre riesgo cardiovascular (ERICA).

Técnica de recolección de datos: se realizó tabulación en Excel de la información recolectada y luego con paquete estadístico SPSS-V23; hallazgos que fueron representados en cuadros y figuras para análisis uni y bivariado de tipo descriptivo, para resignificar los datos e interpretar los resultados y encontrar relaciones entre las variables del estudio.

\section{RESULTADOS}

El Cuadro $\mathrm{N}^{\circ} 1$ muestra que con relación a IMC hay un sobrepeso con RCV de $75 \%$, para RCV alto $25 \%$, y con relación a obesidad un RCV bajo de $84 \%$ y $16 \%$ de RCV alto, lo cual proyectado a 10 años implicaría un alto riesgo de diabetes y como consecuencia riesgo cardiovascular; asociado a una baja actividad física (caminar al lugar de trabajo) y hábitos nocivos de tabaco, que está relacionado con la actividad económica agrícola de la comunidad y consumo de alcohol (fin de semana) pero que por su cantidad constituye un riesgo. 


\section{Cuadro 1.}

RCV general en la población estudio, junio 2018.

\begin{tabular}{cccc}
\hline \multirow{2}{*}{ Variables } & Crierios\% & $\begin{array}{c}\text { RCV moderado } \\
\text { alto }\end{array}$ & \% RCV bajo \\
\hline Hábitos nocivo: & Alcohol $>3$ U/día & 36 & 64 \\
& Tabaco $>10 /$ día & 8 & 92 \\
\hline Actividad física & Si & 10 & 90 \\
(30 minutos actividad & No & 90 & 10 \\
moderada) & & & 90 \\
\hline \multirow{2}{*}{ Clasificación según IMC } & Infrapeso & 10 & 90 \\
& Normopeso & 10 & 75 \\
\hline Riesgo de diabetes & Sobrepeso & 25 & 84 \\
(test Findrisc) & Obesidad & 16 & 75 \\
& & 8 & 0 \\
\hline
\end{tabular}

Fuente: base de datos población estudio

Basados en la información obtenida del Documento Nacional Hábitos y Prácticas Alimentarias (13), en Colombia y específicamente a nivel de Risaralda, existe la cultura de comprar los alimentos ultra procesados (listos para consumir) y procesados (listos para preparar); lo que concuerda con lo dispuesto en las Guías Alimentarias basadas en alimentos para la población colombiana (14), la dieta de la Región Andina es de consumo excesivo en calorías, bajo consumo de frutas, leguminosas y vegetales.

Como se observa en el cuadro $\mathrm{N}^{\circ} 2$, el consumo de agua se encuentra insuficiente con un RCV bajo de $65 \%$ y un RCV de moderado a severo del $35 \%$, porque no cumple las mínimas recomendados dadas por la OMS, representado básicamente por bebidas diferentes al agua como agua de panela, agua jugo y mazamorra entre otros. En cuanto a consumo de alimentos varios, se encontró que es ocasional con un $60 \%$ para RCV bajo y $40 \%$ para RCV alto, evidenciado en el consumo de bebidas como gaseosas, jugos procesados, que constituyen un riesgo, principalmente para la población joven. 


\section{Cuadro 2.}

RCV según hábitos alimentarios en la población estudio, junio 2018.

\begin{tabular}{|c|c|c|c|}
\hline Variables & Crierios & $\begin{array}{l}\mathrm{RCV} \\
\text { \% RCV moderado-alto } \\
\text { muy alto }\end{array}$ & \% RCV bajo \\
\hline Consumo frutas día (3) & $\begin{array}{l}\text { Insuficiente } \\
\text { Deficiente } \\
\text { Suficiente }\end{array}$ & $\begin{array}{l}22 \\
45 \\
20\end{array}$ & $\begin{array}{l}78 \\
55 \\
80\end{array}$ \\
\hline Consumo de verduras día (2) & $\begin{array}{l}\text { Insuficiente } \\
\text { Deficiente } \\
\text { Suficiente }\end{array}$ & $\begin{array}{l}25 \\
35 \\
10\end{array}$ & \\
\hline Consumo de agua día (8) & $\begin{array}{l}\text { Insuficiente } \\
\text { Deficiente } \\
\text { Suficiente }\end{array}$ & $\begin{array}{l}35 \\
40 \\
40\end{array}$ & $\begin{array}{l}65 \\
60 \\
60\end{array}$ \\
\hline $\begin{array}{l}\text { Consumo alimentos procesados y } \\
\text { ultra procesados (perfiles nutrientes } \\
\text { OPS/OMS 2016). Azúcar }<10 \% \text { ICT, } \\
<5 \text { gr/día/sal) }\end{array}$ & $\begin{array}{c}\text { No consumo } \\
\text { Ocasional } \\
\text { Frecuente }\end{array}$ & $\begin{array}{c}5 \\
36 \\
78\end{array}$ & $\frac{95}{22}$ \\
\hline $\begin{array}{l}\text { Consumo alimentos varios } \\
\text { (bebidas azucaradas y energéticas) }\end{array}$ & $\begin{array}{c}\text { No consumo } \\
\text { Ocasional } \\
\text { Frecuente }\end{array}$ & $\begin{array}{l}12 \\
40 \\
22\end{array}$ & $\begin{array}{l}88 \\
60 \\
78\end{array}$ \\
\hline
\end{tabular}

Fuente: base de datos población estudio $(n=186)$

\section{DISCUSIÓN}

Los hallazgos de esta investigación confirman que la población estudiada no cumple con las recomendaciones de la OMS, en cuanto al consumo de frutas, verduras y agua, la actividad física y otros hábitos, convirtiéndola en una población con un alto RCV, pues está demostrado que los hábitos alimentarios están estrechamente relacionados con el peso y la tendencia a diferentes estados de salud, principalmente en niños, cuya alimentación depende de las rutinas alimenticias de los adultos cuidadores y de los alimentos que encuentren en la escuela (15).

Por otra parte, llama la atención la falta de actividad física y el sedentarismo en la población del corregimiento de La Florida, ya que este es un referente turístico, donde específicamente se practica ciclismo y senderismo. Hecho que es altamente alarmante si se tiene en cuenta que la actividad física no solo previene las enfermedades cardiovasculares en personas sanas, sino que actúa como coadyuvante en el tratamiento de estas. Como lo señala Amador-Redero y colaboradores (16), la 
población colombiana tiene un nivel de actividad física bajo, respecto a su consumo calórico, lo que significa que el ejercicio no parece ser un hábito extendido entre los colombianos, a pesar de sus beneficios.

En cuento al hábito de fumar, este estudio evidencia un consumo de tabaco de más de 10 cigarrillos por día, aspecto que la convicción científica relaciona con el aumento del RCV, incluso en personas con tabaquismo pasivo, o con hábitos alimentarios inadecuados, porque en general en Colombia no se consumen aquellos alimentos que retrasan la absorción de grasas, colesterol y glucosa, como las frutas, vegetales, hortalizas y el pescado, o bien, los componentes del cigarrillo interfieren en la absorción de los nutrientes (17).

En este sentido, la lucha contra las enfermedades cardiovasculares requiere una respuesta multisectorial coordinada, que abarque tanto políticas de salud como estrategias de intervención con participación comunitaria y ciudadana y con el acompañamiento de diferentes sectores para el empoderamiento y sensibilización sobre hábitos alimenticios, según guías alimentarias; porque definitivamente los hábitos alimentarios que culturalmente existen en Colombia, se relacionan con un aumento del riesgo cardiovascular en la población, y esto es el reflejo de los datos de morbimortalidad del país y la región, por enfermedad cardiovascular y obesidad.

\section{CONCLUSIONES}

- En virtud de estos hallazgos, se señala que es urgente empoderar y sensibilizar las comunidades sobre hábitos alimentarios, incluso empezando desde la escuela, ya que estos se adquieren a muy temprana edad y son enseñados desde el hogar.

- Las medidas de prevención cardiovascular deben empezar por cambios en el estilo de vida por parte de las personas, sin embargo, este estudio encontró una tendencia hacia aquellos alimentos que se asocian con un mayor RCV, reforzado por la falta de actividad física, tabaquismo, entre otros; razones que llevan a pensar en la necesidad de fomentar la salud cardiovascular desde la comunidad. 
1. OPS/OMS y Centro Latinoamericano y del Caribe de información en ciencias de la salud [internet]. Biblioteca virtual en salud. Descriptores en ciencias de la salud [consultado 2019-11-06]. Disponible en:

http://decs.bvs.br/cgi-bin/wxis1660.exe/decsserver/?IsisScript=../cgibin/decsserver/decsserver.xis\&previous_page=homepage\&task=exact term\&interface_language=e\&search_language=e\&search exp $=$ Enfermedades $\% 20$ Cardiovasculares

2. Organización Mundial de la Salud (OMS). Organización Panamericana de la Salud (OPS). [Internet]. Estado de salud en la población. Prevención y control de las enfermedades no transmisibles. 2017. [citado 2019-01-06]. Disponible en:https://www.paho.org/salud-en-las-americas-2017/?post_t_es=prevenciony-control-de-las-enfermedades-no-transmisibles\&lang=es

3. Organización Mundial de la Salud (OMS). [Internet]. Informe sobre la situación mundial de las enfermedades no transmisibles .2014. [citado 2019-01-06]. Disponible en: http://apps.who.int/iris/bitstream/handle/10665/149296/WHO NMH_NVI_15.1_spa.pdf;jsessionid=0B0EA80539CC8B1114124429D823BB CC? sequence $=1$

4. Ministerio de Salud y Protección Social (MinSalud). Gobierno de Colombia [Internet]. Análisis de la situación de salud Colombia 2015 (ASIS). Bogotá 2015. Pág. 140-146. Disponible en: https://www.minsalud.gov.co/sites/rid/Lists/ BibliotecaDigital/RIDE/VS/ED/PSP/asis-2015.pdf

5. Ministerio de Salud y Protección Social (Minsalud). Gobierno de Colombia [Internet]. Consejo Nacional de Política Económica Social. Política Nacional de Seguridad Alimentaria y Nutricional (PSAN). Documento CONPES Social 113. Bogotá 2008. [citado 2018-06-06]. Disponible en: http://www.sipi.siteal. iipe.unesco.org/sites/default/files/sipi_normativa/colombia_politica_nacional_ de seguridad_alimentaria y nutricional_psan_2008.pdf

6. Ministerio de Protección Social, Gobierno de Colombia [Internet]. Ley1355 de 2009. Diario Oficial No. 47.502 de 14 de octubre de 2009 [citado 2018-02-06]. Disponible en: http://www.redconsumidor.gov.co/descargar.php?idFile=11024

7. Ministerio de Salud y Protección Social (MinSalud). Política de Atención Integral en Salud. Un sistema de salud al servicio de la gente. Bogotá D.C. 2016 [citado 2018-02-06]. Disponible en: https://www.minsalud.gov.co/sites/rid/ Lists/BibliotecaDigital/RIDE/DE/modelo-pais-2016.pdf

8. Ministerio de Salud y Protección Social, Gobierno de Colombia [Internet]. RIAS para la población con riesgo o alteraciones cardio-cerebro-vascular-metabólicas manifiestas, y RIAS específicas para hipertensión arterial-HTA, diabetes mellitus-DM y obesidad. Política de Atención en Salud. 1 carpeta comprimida en zip. 2016. Bogotá D.C. [citado 2018-02-06]. Disponible en: https://www. minsalud.gov.co/sites/rid/paginas/freesearchresults.asp $\mathrm{x}$. $=\& \mathrm{k}=$ cardio

9. Ministerio de Salud y Protección, Gobierno de Colombia [Internet]. Encuesta Nacional de Situación Nutricional (ENSIN). Bogotá D.C. [citado 2019-01-06]. Disponible en: http://www.prosperidadsocial.gov.co/temporales/Encuesta\%20 Nacional $\% 20 \mathrm{de} \% 201 \mathrm{a} \% 20$ Situacio $\% \mathrm{CC} \% 81 \mathrm{n} \% 20$ Nutricional $\% 20-\% 20$ ENSIN\%202015.pdf

10. Fondo Colombiano de Enfermedades de Alto Costo. cuenta de alto costo (CAC) [Internet]. Situación de la enfermedad renal crónica, la hipertensión arterial y la diabetes mellitus en Colombia 2017. Bogotá 2018. [consultada febrero 2018]. 
Disponible en: https://cuentadealtocosto.org/site/images/Publicaciones/2018/ Libro_Situacion_ERC_en_Colombia_2017.pdf

11. Instituto Colombiano de Bienestar Familiar [Internet]. Caracterización y composición nutricional de 18 preparaciones tradicionales en la población colombiana. Bogotá 2017. [consultada agosto 2018]. Disponible en:

https://www.icbf.gov.co/sites/default/files/caracterizacion-composicion-18preparaciones-tradicionales-icbf1_0.pdf

12. Ministerio de Salud y Protección Social (Minsalud), Gobierno de [Internet]. Recomendaciones de ingesta de energía y nutrientes (RIEN) para la población colombiana. Documento técnico. Bogotá 2016. [consultado 2018-03-01]. Disponible en: Disponible en: https:/www.minsalud.gov.co/sites/rid/Lists/ BibliotecaDigital/RIDE/VS/PP/SNA/rien-documento-tecnico.pdf

13. Ministerio de Educación Nacional [internet]. Documento Nacional hábitos y prácticas alimentarias. Hallazgos nacionales a partir del análisis departamental PAE-UNAL. Contrato Interadministrativo No. 918 de 2012. Universidad Nacional de Colombia. [citado 2019-01-06]. Disponible en: https://www. mineducacion.gov.co/1759/articles-336866 archivo pdf UNAL habitos alimentarios.pdf

14.Organización de las Naciones Unidas para la Alimentación y Agricultura (FAO) [internet]. Documento Técnico: Guías Alimentarias basadas en alimentos para la población colombiana mayor de 2 años. Instituto Colombiano de Bienestar Familiar. 2015. [citado 2019-01-06]. Disponible en: https://www.minsalud. gov.co/sites/rid/Lists/BibliotecaDigital/RIDE/VS/PP/SNA/guias-alimentariasbasadas-en-alimentos.pdf

15. Acosta, J.A.; Medrano, G.; Duarte, G.; González, R. Malos hábitos alimentarios $\mathrm{y}$ falta de actividad física principales factores desencadenantes de sobrepeso y obesidad en los niños escolares. Rev. CULCyT. [Internet]. 2014 [consultada febrero 2018]; año 11, No. 54 Especial No.1. pág. 80-90. Disponible en: http://erevistas.uacj.mx/ojs/index.php/culcyt/article/download/871/822.

16. 25. Amador-Rodero, E; Montealegre-Esmeral, L; Arrozola-David, M. Espacios para la actividad física en un corregimiento de Colombia. Biociencias 9(2):2734. Universidad Libre. 2014. Barranquilla Colombia. Disponible en: file:///C:/Users/Mar\%C3\%ADa\%20Posada/Downloads/Dialnet-EspaciosParaL aActividadFisicaEnUnCorregimientoDeCo-5976581.pdf

17. Lanas, F; Serón, K.P. Rol del tabaquismo en el riesgo cardiovascular global [internet]. Rev. Med. Clin. Condes. 2012; 2386):699-700. Temuco, Chile. [citado 2019-01-06]. Disponible en:

http://www.elsevier.es/es-revista-revista-medica-clinica-las-condes-202-pdf$\underline{\mathrm{S} 0716864012703711 \mathrm{~m}}$ 